\title{
Estenosis crítica de conducto hepático común por anillo vascular: reporte de un caso
}

\author{
I. García ・ R. Muñoz • M. Guelfand
}

\begin{abstract}
Common bile duct stricture caused by vascular ring: A case report

Introduction: The most common congenital malformations of the bile duct are biliary atresia and choledochal cyst. In addition, the most common liver anatomical variation is the right hepatic artery aberration. The goal of this study is to characterize a patient with this disease and propose the hepatoduodenal anastomosis as surgical treatment. Case report: One-month-old patient with suspected congenital biliary atresia due to progressive jaundice and acholia since birth. Liver tests consistent with a cholestatic pattern and brain MRI scan consistent with biliary atresia. Periods of decreased bilirubin and sporadic slight pigmentation of depositions were described. The surgical finding was a bile duct stricture due to a vascular ring caused by aberrant right hepatic artery. Resection of bile duct and hepatic-duodenal bypass were performed. The patient evolved satisfactorily from this condition. Conclusion: There are few reports of biliary obstruction due to vascular malformations. It is important to keep in mind that not all neonatal jaundice episodes are caused by biliary atresia or choledochal cyst. The clinical course, laboratory tests and imaging should be considered and in the case of suspicion, further exploration should take place.

(Key words: Biliary stenosis, vascular ring, hepato-duodenal anastomosis, biliary atresia).

Rev Chil Pediatr 2014; 85 (5): 594-598
\end{abstract}

\section{RESUMEN}

Introducción: Las malformaciones congénitas de la vía biliar más frecuentes son la atresia de vías biliares y quiste de colédoco. Por otro lado, la variante anatómica hepática más común es la aberración de la arteria hepática derecha. El objetivo es caracterizar un paciente portador de esta patología y plantear la hepato-duodeno anastomosis como tratamiento quirúrgico. Caso clínico: Paciente de 1 mes de edad, con sospecha de Atresia de Vía Biliar congénita por ictericia progresiva y acolia desde recién nacido. Pruebas hepáticas concordantes con un patrón colestásico y resonancia magnética compatible con atresia de vías biliares. Evolucionó con períodos de descenso de bilirrubina y leve pigmentación, esporádica, de deposiciones. El hallazgo quirúrgico fue una estenosis crítica de vía biliar a nivel del conducto hepático común debido a un anillo vascular por una arteria hepática derecha aberrante. Se realizó una sección de vía biliar y una derivación hepato-duodenal. Evolucionó con una resolución completa de su patología. Conclusión: Existen pocos reportes de obstrucción de vía biliar por malformaciones vasculares. Es importante tener presente que no todas las ictericias neonatales son por atresia de vías biliares o quiste de colédoco. Se debe considerar la evolución clínica, laboratorio e imágenes, y si existen sospechas, explorar.

(Palabras clave: Estenosis vía biliar, anillo vascular, hepático-duodeno anastomosis, atresia vía biliar).

Rev Chil Pediatr 2014; 85 (5): 594-598

Recibido el 07 de junio de 2014, última versión aceptada el 01 de octubre de 2014.

Isidora García Larraín ( $\varangle)$, Miguel Guelfand Chaimovich

Cirujano Pediátrico, Hospital Exequiel González Cortés, Santiago, Chile.

E-mail:

Rosa Muñoz Villanueva

Residente de Cirugía Pediátrica, Hospital Exequiel González Cortés, Santiago, Chile. 


\section{Introducción}

La anatomía vascular de la irrigación hepática es muy variable. Las variantes del esquema dominante, en que el hígado recibe su total irrigación de la arteria hepática desde el tronco celíaco, ocurren en 25 a $75 \%$ de los $\operatorname{casos}^{1}$. La irrigación hepática clásica fue descrita por Covey et al, Koops et al y Michels. Ellos describen que desde la aorta se origina el tronco celíaco y se bifurca en arteria esplénica, hepática común y gástrica izquierda, la arteria hepática común corre por el borde anterosuperior del páncreas originando de ella la arteria gastroduodenal, luego de esto se llama hepática propia, que luego se divide en hepática derecha e izquierda.

La variación más común descrita es el reemplazo de la arteria hepática derecha, que se origina de la arteria mesentérica superior en un 11 a $21 \%$. Se han descrito arterias hepáticas derechas e izquierdas accesorias en un 0,8 a $8 \%{ }^{2}$.

Por otro lado, las malformaciones congénitas más frecuentes de la vía biliar son la atresia de vías biliares y el quiste de colédoco. Muchas enfermedades, benignas y malignas pueden causar obstrucción de la vía biliar extra hepática y la complicación más seria de la obstrucción es la colangitis, llevando en ocasiones a una descompresión de urgencia. Son frecuentes las variaciones a nivel de la vía biliar pero son pocos los casos publicados en que la obstrucción está dada por vasos arteriales ${ }^{3}$.

Se han descritos casos de obstrucción, la mayoría en adultos, dada por arterias aberrantes, la mayoría idiopática, casos de ictericia por compresión por la arteria hepática derecha, por aneurisma de la arteria hepática y por transformación cavernomatosa ${ }^{4}$.

El objetivo de la publicación es caracterizar un paciente portador de esta la patología y plantear la hepato-duodeno anastomosis como tratamiento quirúrgico de la misma.

Presentamos un caso de una niña de 1 mes de vida que presenta una estenosis crítica de vía biliar congénita secundaria a una malformación de la arteria hepática derecha, específicamente un anillo vascular.

\section{Caso clínico}

Paciente femenino, previamente sano, con antecedentes de embarazo controlado, nacido a término 38 semanas, pequeña para la edad gestacional $(2.670 \mathrm{~kg})$. Consultó a los 47 días de vida por cuadro de 20 días de evolución caracterizado por ictericia progresiva y coluria, con buen incremento de peso, siendo derivada al hospital regional para estudio.

Exámenes con patrón colestásico, en los cuales destacaba aumento de bilirrubina total (BT), a expensas de la bilirrubina directa (BD) $(12,75 \mathrm{mg} / \mathrm{dl}-10,18 \mathrm{mg} / \mathrm{dl})$ y pruebas hepáticas elevadas, sobre todo la gamma glutamil transpeptidasa (GGT) y la fosfatasa alcalina (FA).

En Hospital regional se sospechó hepatitis viral, y se tomó serología para hepatitis A, B y $\mathrm{C}$ que resultaron negativas. Evolucionó favorablemente pero se constataron deposiciones acólicas. Se derivó a gastroenterología previos exámenes de control que mantenían patrón colestásico con elevación de la BD y de las transaminasas: BT 10,58, BD 7,88, GOT 204, GPT 109, GGT 550.

A los 52 días de vida se le realizó una ecografía abdominal que mostró dilatación arrosariada de vía biliar intrahepática y vesícula biliar presente pero colapsada a pesar del ayuno.

Por la presencia de la vesícula y la gran dilatación de vía biliar intrahepática, se decidió diferir la biopsia hepática y se realizó una colangio-resonancia. Ésta mostró un hígado normal con parénquima conservado, leve dilatación de ductos biliares intrahepáticos hasta nivel de su confluencia, hacia distal no se logró visualizar vía biliar extrahepática.

Con el resultado de la colangio-resonancia se sospechó atresia de vía biliar y se decidió cirugía de derivación bilio-digestiva (Kasai). Llamaba la atención que en exámenes seriados la bilirrubina había ido en disminución al igual que la ictericia. Por presencia de deposiciones acólicas se decidió exploración quirúrgica.

Durante la cirugía se visualizó superficie hepática lisa, hígado congestivo (confirmado por biopsia), vesícula biliar presente, con lumen y bilis en su interior. Se realizó colangiografía intraoperatoria donde se visualizó una zona estenótica crítica a nivel del conducto he- 


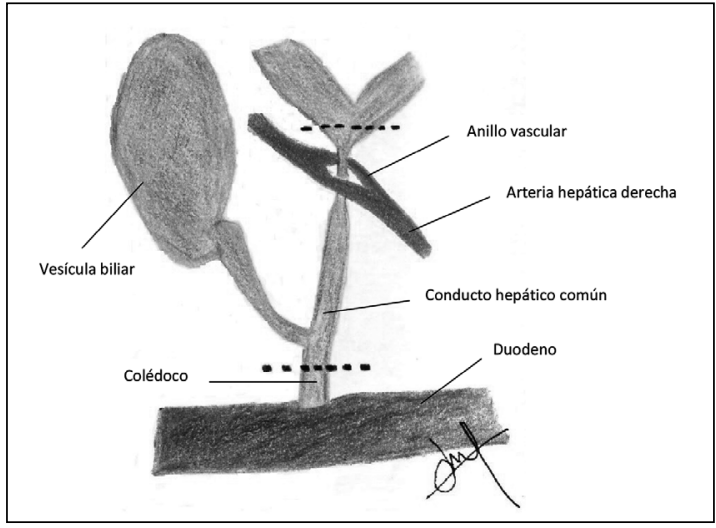

Figura 1. Estenosis por anillo vascular.

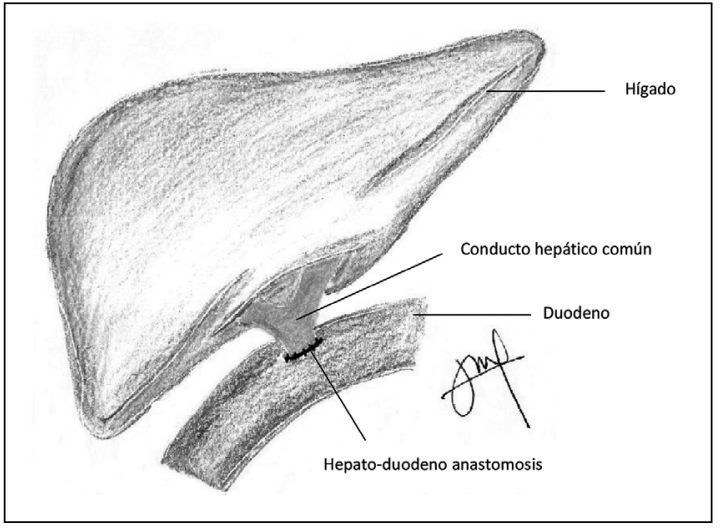

Figura 2. Hepato-duodeno anastomosis.

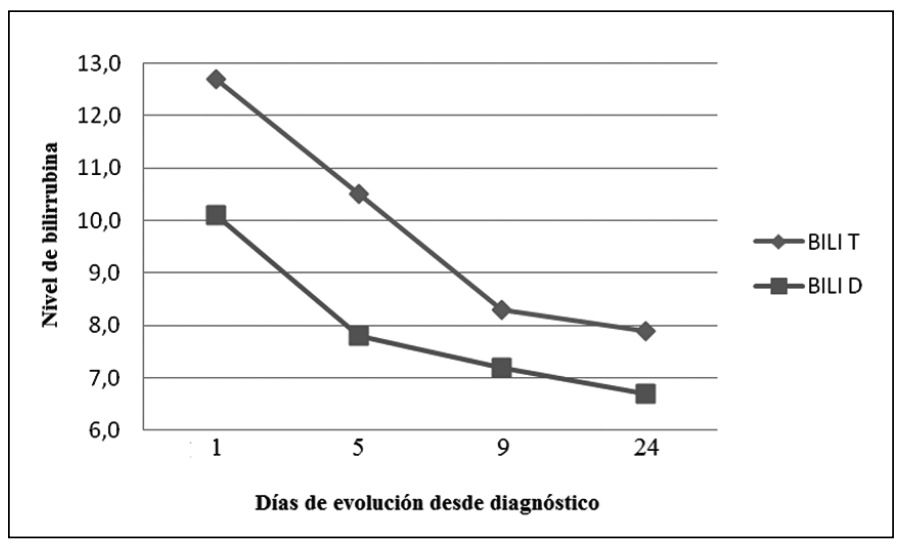

Figura 3. Resumen de evolución de bilirrubina. BILI T: Bilirrubina total. BILI D: Bilirrubina directa. pático común logrando paso de contraste hacia proximal y distal (intra y extra hepático), con vía biliar intrahepática dilatada.

Se realizó colecistectomía y disección de conducto hepático común demostrando una estenosis a nivel de éste, causado por un anillo vascular proveniente de arteria hepática derecha (figura 1). Se seccionó la vía biliar a nivel proximal de la estenosis realizando una hepático-duodeno anastomosis (figura 2). Se dejó drenaje abdominal y sonda nasogástrica instalada.

La paciente evolucionó favorablemente, con débito en disminución por el drenaje retirándose al 6 día y alimentándose a los 4 días. Se dio de alta a los 8 días post operatorio con pruebas hepáticas en disminución (BT 3,98, BD 3,46, GOT 266, GPT 134, FA 431, GGT
586) (figura 3). En controles ambulatorios la paciente se encontraba asintomática, con buena tolerancia oral, deposiciones teñidas y exámenes hepáticos y generales normalizados.

\section{Discusión}

La irrigación hepática es una anatomía muy variada, siendo las variaciones más comunes el reemplazo y las arterias accesorias de la hepática derecha ${ }^{5}$. Por otro lado, las malformaciones más comunes de la vía biliar son el quiste de colédoco y la atresia de vías biliares, por lo que en niños pequeños son los primeros diagnósticos a descartar frente a una ictericia neonatal de predominio directo ${ }^{6}$. La incidencia del quiste de colédoco en los países occi- 
dentales varía entre 1 en 100.000 a 150.000 , siendo tres veces más alta en países asiáticos? La incidencia de la atresia de vías biliares reportada a nivel mundial es de alrededor de 1 cada 9.000 a 12.000 recién nacidos vivos, sin embargo, se presenta como la principal causa de ictericia de origen extrahepático y así mismo de trasplante hepático ${ }^{8}$.

Son pocos los casos descritos de estenosis de vía biliar congénita. Existen 4 casos publicados de obstrucción de vía biliar por anillo vascular, todos en adultos: uno en que se describe el reemplazo de la arteria hepática derecha por 2 arterias segmentarias que forman un anillo vascular alrededor del conducto hepático común pero sin ictericia ${ }^{3}$, el segundo de una mujer 68 años que se hospitaliza por un cuadro de colangitis, y dentro de su estudio se determina la presencia de una dilatación intra y extra hepática, secundaria a un anillo vascular de la arteria hepática común, proveniente de la arteria mesentérica superior ${ }^{3}$, otro de un hombre 63 años con ictericia colestásica causada por anillo vascular esclerótico de la arteria hepática derecha ${ }^{9}$ y el cuarto es un caso descrito en una revista de anatomía patológica como hallazgo en un cadáver ${ }^{10}$.

Tanto estos casos, como otros casos de estenosis idiopática de vía biliar se resolvieron con una derivación biliodigestiva en Y de Roux ${ }^{4,11}$.

Este caso fue resuelto con la sección de la vía biliar a nivel del conducto hepático común pre estenosis y una anastomosis término-lateral hepático-duodenal por anterior al anillo vascular.

Esta anastomosis presenta ventajas frente a la tradicional hepático-yeyuno anastomosis en Y de Roux, tales como: a) se realiza sólo 1 anastomosis; b) los pacientes son alimentados más precozmente; c) menor estadía hospitalaria y d) mejor accesibilidad endoscópica a la anastomosis para el seguimiento a largo plazo. Es más, otros trabajos publicados no han reportado reflujo ni diferencias estadísticas en síntomas post operatorios comparando las 2 técnicas ${ }^{15}$.

Esta anastomosis hepático-duodenal, es ampliamente discutida ya que hay autores que refieren que se produciría un reflujo básico al estomago y vía biliar provocando inicialmente gastritis, dispepsia, disfagia, colangitis y dolores abdominales, y a largo plazo colangiocarcinoma. No se ha demostrado diferencia significativa en estudios histológicos comparativos $^{12}$. Las ventajas de realizar una hepático-duodeno anastomosis reportadas refieren que sería más fisiológica, técnicamente más fácil y menor tiempo operatorio, menos obstrucciones intestinales, menor filtración de la anastomosis y menor tasa de úlcera péptica ${ }^{13}$. No hay estudios que comparen esta técnica vía laparoscópica versus abierta.

Todani et al, los primeros en publicar resultados comparativos entre hepático-duodeno anastomosis e Y de Roux, dejaron de realizar esta anastomosis cuando encontraron en un paciente un carcinoma de via biliar 19 años después de la cirugía. Ellos postulan que el reflujo duodenal a la vía biliar, incluyendo enzimas pancreáticas, alterarían la histología aumentando el riesgo de cáncer ${ }^{14}$.

\section{Conclusión}

Nos parece importante destacar que no todas las ictericias neonatales son por atresia de vías biliares o quiste de colédoco. Se debe considerar la evolución clínica, laboratorio e imágenes, y si existen sospechas explorar. Además concluimos en este caso la hepático-duodeno anastomosis fue una resolución efectiva y segura para una obstrucción completa de vía biliar.

Potenciales conflictos de interés: Este trabajo cumple con los requisitos sobre consentimiento/asentimiento informado, comité de ética, financiamiento, estudios animales y sobre la ausencia de conflictos de intereses según corresponda.

\section{Referencias}

1.- Hiatt JR, Gabbay J, Busuttil RW: Surgical Anatomy of the Hepatic Arteries in 1000 Cases. Ann Surg 1994; 220 (1): 50-2.

2.- Shukla PJ, Barreto SG, Kulkarni A, Nagarajan G, Fingerhut A: Vascular Anomalies Encountered During Pancreatoduodenectomy: Do They Influence Outcomes? Ann Surg Oncol 2010; 17 (1): 186-93. 
3.- Bilanovic D, Zdravkovic D, Toskovic B, et al: Obstructive jaundice and cholangitis caused by an arterial ring of the proper hepatic artery around the common bile duct. Med Sci Monit 2011; 17 (8): CS91-3.

4.- Mazzariello RM, Novas OL, Salinas J: Estenosis congénitas e idiopáticas de las vías biliares. Rev Argent Cir 1993; 65 (1/2): 7-11.

5.- De Cecco CN, Ferrari R, Rengo M, Paolantonio P, Vecchietti $F$, Laghi A: Anatomic variations of the hepatic arteries in 250 patients studied with 64-row CT angiography. Eur Radiol 2009; 19 (11): 2765-70.

6.- Todani T, Watanabe Y, Toki A, Morotomi Y: Classification of congenital biliary cystic disease: special reference to type Ic and IVA cysts with primary ductal stricture. J Hepatobiliary Pancreat Surg 2003; 10 (5): 340-4.

7.- Pereira N, Benavides J, Espinoza C, Rostión CG: Quiste de colédoco en pediatría: Una Revisión de la Literatura. Rev Ped Elec [en línea] 2007: Vol 4, N³.

8.- Benavides J, Espinoza C, Pereira N, Rostion CG: Atresia de vías biliares en pediatría: Una Revisión de la Literatura. Rev Ped Elec [en línea] 2008; Vol 5, №3.

9.- Takehara H, Komi N, Okada A, et al: Unusual cases of benign stricture of the biliary tract. Tokushima J Exp
Med 1992; 39 (3-4): 135-43.

10.- Dusanović AM, Ignjatović DM, Knezević PA, Todorović $M$, Krgović KL, Filipović BR: Arterial ring encircling the bile duct with coexisting anomalous liver irrigation. Dig Surg 1999; 16 (1): 72-5.

11.- Chapoy PR, Kendall RS, Fonkalsrud E, Ament ME: Congenital stricture of the common hepatic duct: an unusual case without jaundice. Gastroenterology 1981; 80 (2): $380-3$

12.- Takada K, Hamada Y, Watanabe Y, Tanano A, Tokuhara $K$, Kamiyama $Y$ : Duodenogastric reflux following biliary reconstruction after excision of choledochal cyst. Pediatr Surg Int 2005; 21: 1-4.

13.- Davenport M, Betalli P, D'Antiga L, et al: The spectrum of surgical jaundice in infancy. J Pediatr Surg 2003; 38: 1471-9.

14.- Todani T, Watanabe Y, Mizuguchi T, et al: Hepaticoduodenostomy at the hepatic hilum after excision of choledochal cyst. Am J Surg 1981; 142: 584-7.

15.- Santore M, Behar B, Blinman T, et al: Hepaticoduodenostomy $v s$ hepaticojejunostomy for reconstruction after resection of choledochal cyst. Journal of Pediatric Surgery $2011 ; 46:$ 209-13. 